\title{
Age-dependent prevalence of 14 high-risk HPV types in the Netherlands: implications for prophylactic vaccination and
}

\section{screening}

\author{
VMH Coupé*,', J Berkhof', NWJ Bulkmans², PJF Snijders² and CJLM Meijer² \\ 'Department of Clinical Epidemiology and Biostatistics, VU University Medical Centre, PO Box 7057, Amsterdam 1007 MB, The Netherlands; \\ ${ }^{2}$ Department of Pathology, VU University Medical Centre, PO Box 7057, Amsterdam 1007 MB, The Netherlands
}

We determined the prevalence of type-specific hrHPV infections in the Netherlands on cervical scrapes of 45362 women aged I865 years. The overall hrHPV prevalence peaked at the age of 22 with peak prevalence of $24 \%$. Each of the 14 hrHPV types decreased significantly with age ( $P$-values between 0.0009 and 0.03 ). The proportion of HPV 16 in hrHPV-positive infections also decreased with age $(\mathrm{OR}=0.76$ ( 10 -year scale), 95\% $\mathrm{Cl}=0.67-0.85)$, and a similar trend was observed for HPVI 6 when selecting hrHPV-positive women with cervical intraepithelial neoplasia grade 2 or worse $(\mathrm{CIN} 2+)(\mathrm{OR}=0.76,95 \% \mathrm{Cl}=0.56-1.0 \mathrm{I})$. In women eligible for routine screening (age 29-6I years) with confirmed CIN2 +, 65\% was infected with HPVI6 and/or HPVI8. When HPVI6/I8positive infections in women eligible for routine screening were discarded, the positive predictive value of cytology for the detection of CIN2 + decreased from 27 to 15\%, the positive predictive value of hrHPV testing decreased from 26 to I5\%, and the predictive value of a double-positive test (positive HPV test and a positive cytology) decreased from 54 to $41 \%$. In women vaccinated against HPVI6/I8, screening remains important to detect cervical lesions caused by non-HPVI6/I8 types. To maintain a high-positive predictive value, screening algorithms must be carefully re-evaluated with regard to the screening modalities and length of the screening interval.

British Journal of Cancer (2008) 98, 646-65I. doi:I0.1038/sj.bjc.6604I62 www.bjcancer.com

Published online 8 January 2008

(C) 2008 Cancer Research UK

Keywords: human papillomavirus; age-dependent prevalence; vaccination; screening

Infection with high-risk human papillomavirus (hrHPV) is the necessary cause of cervical cancer (Munoz et al, 2003). Prophylactic vaccines are now available that are effective against incident and persistent HPV16 and 18 infections (Koutsky et al, 2002; Munoz et al, 2003; Harper et al, 2006; Villa et al, 2006). Mass vaccination can have a substantial impact on the cervical cancer incidence, even in developed countries where the incidence is already low because of implementation of organised cervical screening. To make a well-judged decision about the future role of both vaccination and screening in cervical cancer prevention, detailed information about type-specific hrHPV distribution is required. Data on hrHPV prevalence and type distribution in the Netherlands have been reported previously, but only for a relatively small cohort of 3305 women (Jacobs et al, 2000a; Clifford et al, 2005). To obtain reliable estimates of the hrHPV type distribution in relation to age, both for women with normal and for women with abnormal cytology, data from a far larger cohort are needed. In this study, we determined the age-dependent prevalence of $14 \mathrm{hrHPV}$ types and the age-dependent type distribution within hrHPV-positive women from a cohort of

*Correspondence: Dr VMH Coupé; E-mail: v.coupe@vumc.nl Received 23 August 2007; revised 16 November 2007; accepted 28 November 2007; published online 8 January 2008
45362 women in the Netherlands aged 18-65 years. The results from the current study give an impression of the potential benefits that can be achieved from HPV16/18 vaccination. They can also be used to gain insight into the effects of partial cross-protection against HPV types 31 and 45 (Harper et al, 2006). Furthermore, the figures will serve as inputs for simulation models in which different vaccination and screening strategies will be compared.

\section{MATERIALS AND METHODS}

\section{Data}

Data from 45362 Dutch women between 18 and 65 years of age were collected. Cohort participants were recruited from GP practices in the larger Amsterdam area between 1999 and 2002. Women between 29 and 61 years of age $(n=44102)$ participated in the POBASCAM study, a randomised controlled population-based screening trial evaluating the implementation of hrHPV testing in cervical screening (Bulkmans et al, 2004). The POBASCAM trial was approved by both the Medical Ethics Committee of the VU University Medical Center (no. 96/103) and the Ministry of Public Health (VWS no. 328650) and has been registered as an International Standard Randomised Controlled Trial (ISRCTN20781131). Women younger than 29 years $(n=1109)$ or older than 61 years $(n=151)$ obtained a cervical smear for 
opportunistic screening. All women underwent cytological testing as well as hrHPV testing. Women with abnormal cytology or cervical intraepithelial neoplasia (CIN) in the previous 2 years were excluded.

\section{Cytology, hrHPV DNA testing, genotyping}

Conventional cytological smears were collected and read according to the CISOE-A classification used in the Netherlands. Translation into the Bethesda 2001 classification is easy (Bulk et al, 2004). Cytological results were grouped as normal or borderline mild dyskaryosis (BMD), and $>$ BMD. Cervical scrapes were tested for the presence of hrHPV DNA using the consensus GP5 + /6+-PCREIA method, which detects $14 \mathrm{hrHPV}$ types (HPV16, 18, 31, 33, 35, $39,45,51,52,56,58,59,66$, and 68) (Jacobs et al, 1997). hrHPV testing was blinded to the cytological result. hrHPV-positive samples were subjected to hrHPV typing by reverse line blot analysis of PCR products (van den Brule et al, 2002). Of screeningeligible women who participated in the POBASCAM trial (aged 29-61 years), histological results of biopsy specimen taken at colposcopy inspection were included. All cases of CIN were included that were found after the referral smear (baseline smear or repeat smear at 6 or 18 months) and within 3 years after baseline. Cases were classified as CIN 1, 2, or 3 or invasive cancer in the local hospital and were not subjected to revision.

\section{Statistical methods}

Age-specific estimates of the hrHPV prevalence (10-year age groups) were computed for all women and for women with normal cytology. Overall and type-specific hrHPV prevalences were related to age by logistic regression analyses. To obtain smooth functions of age, cubic splines with knots at age 30 and 50 years were used (Wold, 1974). The uncertainty about the estimated splines was represented by $99 \%$ point wise correct confidence intervals. Within the subset of hrHPV-positive women, the effect of age on the type distribution was assessed by logistic regression analyses. In statistical tests, only screening-eligible women (age 29-61 years) were included. Separate analyses were performed for women without confirmed $\mathrm{CIN} 2+$ and for women with confirmed $\mathrm{CIN} 2+$. The analyses were repeated for women with single infections.
With vaccination in mind, particular attention was paid to HPV types $16,18,31$, and 45 . Types 31 and 45 are of interest because vaccination against $16 / 18$ may result in partial immunity for 31 and 45 through cross-protection (Harper et al, 2006). Within the group of screening-eligible hrHPV-positive women, we estimated the type distribution of HPV16, 18, 31, 45 vs other types. Separate calculations were carried out for women without confirmed $\mathrm{CIN} 2+$ and women with confirmed CIN2 + . This was carried out by hierarchically assigning women to (1) HPV16, (2) HPV18 without the presence of HPV16, (3) HPV31 or 45 without HPV16 or 18 , or (4) none of the HPV types $16,18,31$, or 45 . This means that, for example, assignment to HPV16 was carried out independently of whether a woman was simultaneously infected with other HPV types. Analyses were repeated for women with single infections.

Vaccination against HPV types 16 and 18 will affect current screening practice, because a decrease in the prevalence of highgrade CIN is expected to lead to a decrease in the positive predictive value of a screening instrument. To gain insight into the potential effects of vaccination on the predictive value of a positive screening test, we computed the positive predictive values for detection of CIN2 + for women without HPV16 and 18. We computed separate predictive values of positive cytology, a positive HPV DNA test, and a double-positive test (positive HPV test and a positive cytology). Notably, the double-positive test result is a prerequisite for colposcopy referral in a screening setting where cytology is used as a triage tool in HPVpositive women. The analyses were repeated for women without HPV16, 18, 31, and 45. The positive predictive values were adjusted for women who did not attend repeat screening by Kaplan-Meier estimation.

\section{RESULTS}

Table 1 shows the age-specific estimates of the hrHPV prevalences, for all women combined and for women with normal cytology. Table 1 shows the number of women in each age group who are infected with each of the $14 \mathrm{HPV}$ types. The overall hrHPV prevalence was $5.6 \%$. In women with normal cytology, the hrHPV prevalence was $4.0 \%$. HPV 16 was the most common type $(1.8 \%$ in all women, $1.1 \%$ in normal cytology), followed by types 31 and 18 . The hrHPV prevalence in all women aged 29-61 years decreased

Table I The hrHPV prevalences in I0-year age-groups, for all women combined $(n=45362)$ and for women with normal cytology $(n=43737)$

\begin{tabular}{|c|c|c|c|c|c|c|c|c|c|c|c|c|c|c|c|c|}
\hline $\begin{array}{l}\text { Age- } \\
\text { group }\end{array}$ & $n$ & $\begin{array}{c}\text { Total } \\
\text { hrHPV (\%) }\end{array}$ & HPVI 6 & HPVI 8 & HPV3 I & HPV33 & HPV35 & HPV39 & HPV45 & HPV5 I & HPV52 & HPV56 & HPV58 & HPV59 & HPV66 & HPV68 \\
\hline \multicolumn{17}{|l|}{ All women } \\
\hline $18-24$ & 482 & $102(2 \mid .2)$ & 36 & | | & 15 & 6 & 4 & 6 & 3 & 13 & 8 & 14 & 4 & 5 & 14 & 0 \\
\hline $25-34$ & 10828 & $|16|(10.7)$ & 419 & 127 & 174 & 76 & 48 & 67 & 78 & 93 & 93 & 79 & 65 & 26 & 76 & 17 \\
\hline $35-44$ & 15303 & $753(4.9)$ & 235 & 60 & 112 & 48 & 39 & 39 & 65 & 46 & 36 & 52 & 53 & 14 & 30 & 15 \\
\hline $45-54$ & II 556 & $321(2.8)$ & 80 & 37 & 36 & 18 & 12 & 8 & 22 & 21 & 19 & 34 & 16 & 8 & 24 & I \\
\hline $55-65$ & 7193 & $184(2.6)$ & 49 & 14 & 23 & 14 & 10 & 6 & 6 & 15 & 7 & 20 & II & 5 & 10 & 4 \\
\hline Total & 45362 & $252 \mid(5.6)$ & 819 & 249 & 360 & 162 & 113 & 126 & 174 & 188 & 163 & 199 & 149 & 58 & 154 & 37 \\
\hline \multicolumn{17}{|c|}{ Women with normal cytology } \\
\hline $18-24$ & 440 & $80(18.2)$ & 31 & 6 & || & 4 & 4 & 5 & 3 & 9 & 5 & || & 2 & 4 & 9 & 0 \\
\hline $25-34$ & 10238 & $770(7.5)$ & 246 & 81 & 127 & 38 & 31 & 44 & 50 & 62 & 55 & 53 & 41 & 18 & 60 & 9 \\
\hline $35-44$ & $|475|$ & $508(3.4)$ & 132 & 39 & 76 & 31 & 29 & 25 & 48 & 26 & 23 & 39 & 35 & 13 & 19 & | | \\
\hline $45-54$ & | | 239 & $244(2.2)$ & 52 & 26 & 26 & 13 & 7 & 5 & 19 & 10 & 17 & 30 & | | & 8 & 19 & I \\
\hline $55-65$ & 7069 & $156(2.2)$ & 41 & | | & 19 & 11 & 10 & 6 & 6 & 9 & 5 & 20 & 10 & 5 & 10 & 4 \\
\hline Total & 43737 & | $758(4.0)$ & 502 & 163 & 259 & 97 & 81 & 85 & 126 & 116 & 105 & 153 & 99 & 48 & 117 & 25 \\
\hline
\end{tabular}

In each age group, the number of women that is infected with each of the I4 HPV types is shown. 
significantly with age for all hrHPV types ( $P$-values between 0.0009 and 0.03). Significance could also be demonstrated when selecting only those women with normal cytology.

Figure 1 shows the age-dependent overall hrHPV prevalence for all women irrespective of cytological status (Figure 1A) and for women with normal cytology (Figure 1B). The dashed lines represent the lower and upper $99 \%$ confidence bands of the fitted curves. Age-specific estimates (10-year age groups) and $99 \%$ confidence intervals were also included in the figures. Overall hrHPV prevalence peaked at 22 years, with a peak prevalence of $24 \%$. Beyond 45 years, the hrHPV prevalence reached a plateau of $3 \%$. For normal cytology, a similar pattern was observed with a slightly lower peak prevalence of $20 \%$ (Figure 1B). The fitted curves lie within the $99 \%$ confidence intervals of the age-specific estimates. Because only a small sample of young women (18-24 years) was included in the data, wide confidence intervals were found in this age group. Figure 2 shows separately fitted prevalence curves for hrHPV types 16, 18, 31, and 45 in women with normal cytology. Again, the functions were single-peaked with peaks before age 30 years.

Within the set of hrHPV-positive screening-eligible women (age 29-61 years) with a valid HPV typing result, the proportion of HPV16 infections decreased significantly with age (OR $=0.76(10-$ year scale), $95 \% \mathrm{CI}=0.67-0.85, P<0.001)$. For HPV39 and HPV52, a marginal decrease with age was found as well (both $P$-values 0.03$)$. In the subset of women with confirmed CIN2 + , the association between HPV16 and age was in the same direction $(\mathrm{OR}=0.76, \quad 95 \% \quad \mathrm{CI}=0.56-1.01, \quad P=0.06)$. No relationship between hrHPV type and age was observed for any of the other types. The impact of age on the proportion of HPV16 infections in hrHPV-positive women is illustrated in Figure 3. In women with confirmed CIN2 + , the proportion of HPV16 infections decreased
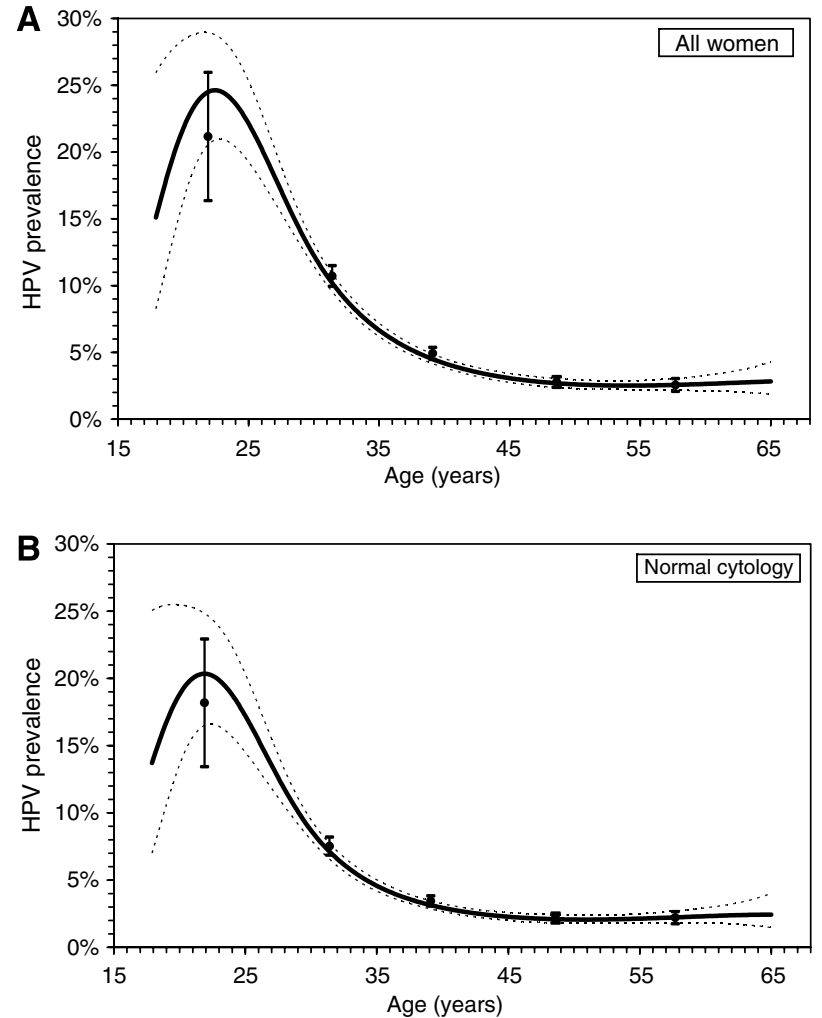

Figure I The relationship between hrHPV prevalence and age for all women $(n=45362) \quad(\mathbf{A})$ and for women with normal cytology $(n=43737)(\mathbf{B})$. from $59 \%$ in women younger than 40 years to $53 \%$ in older women. The results for women with single infections were nearly identical.

In hrHPV-positive women who were eligible for routine screening, 33\% was infected with HPV16, 9\% was infected with HPV18 without 16, and 59\% was infected with HPV16, 18, 31, and/ or 45. HrHPV infections with types other than HPV16, 18, 31, and/ or 45 were found in $41 \%$ of the women. Figure 4 shows the type distribution in screening-eligible hrHPV-positive women with confirmed $\mathrm{CIN} 2+$ and in women without confirmed CIN2 + In women with confirmed CIN2 + , HPV16 and/or HPV18 was found in $65 \%$ of the women. Women with infections other than HPV $16,18,31$, or 45 comprised $24 \%$ of the women with confirmed $\mathrm{CIN} 2+$. The type distribution in women with single infections was very similar.

Figure 5 shows the positive predictive values for detection of CIN2 + lesions in women aged 29-61 years eligible for routine screening, in case screening is carried out by means of cytology, HPV testing or both cytology and HPV testing. The black bars represent the predictive values for the current HPV type distribution. The predictive values of abnormal cytology and a positive hrHPV test for detection of CIN2 + were 27 and $26 \%$, respectively. The predictive value of positive results on both tests was 54\%. When excluding HPV16- and HPV18-positive infections from the data, the predictive values of positive cytology, positive

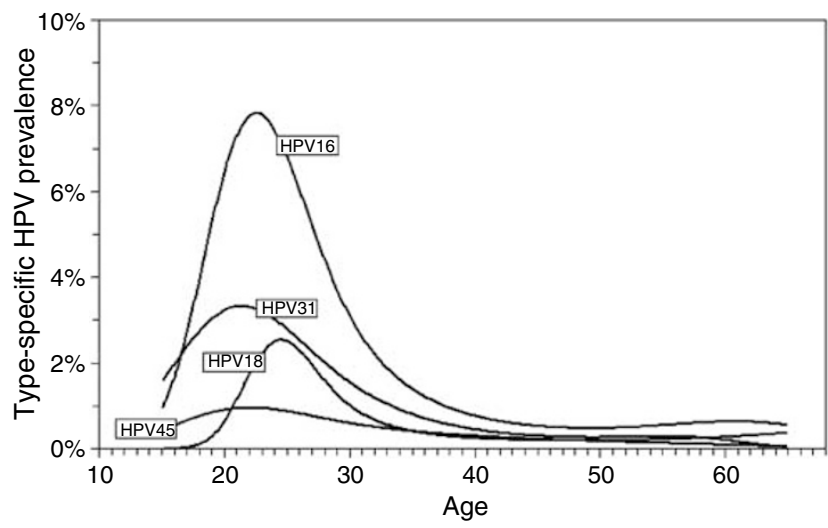

Figure 2 The relationship between the prevalence of HPV types 16, 18 , 31 , and 45 and age for women with normal cytology $(n=43875)$.

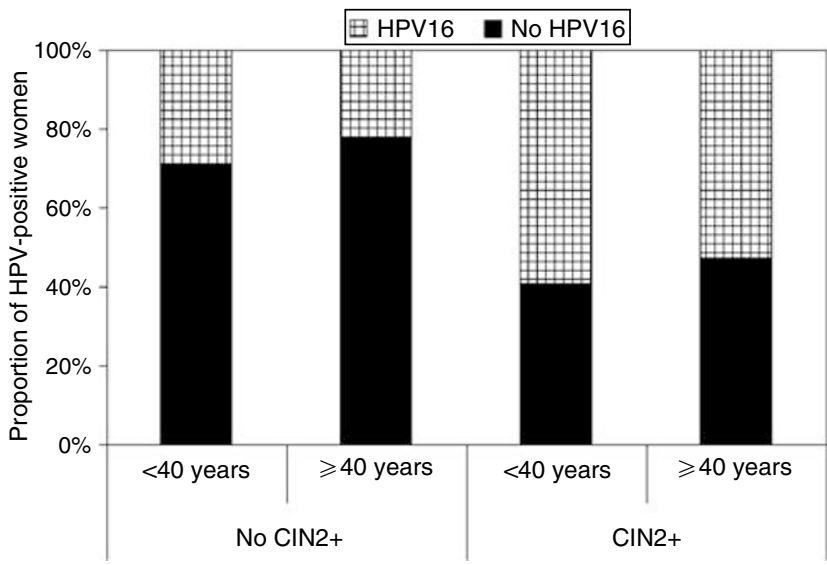

Figure 3 The proportion of HPVI6 in hrHPV infections in women younger than 40 years of age and older women. Results are given for women without confirmed CIN2 + and for women with confirmed $\mathrm{CIN} 2+$. 


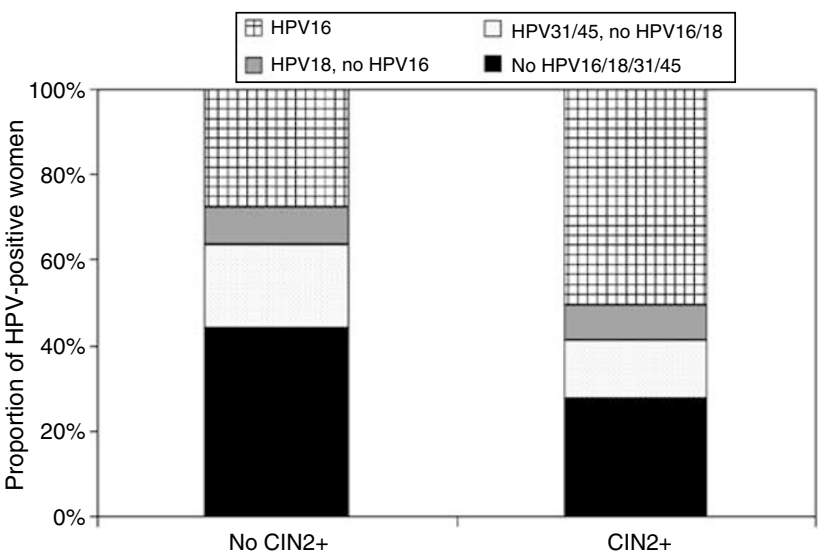

Figure 4 The HPV type distribution in hrHPV-positive women eligible for routine screening. The type distribution is shown separately for women without confirmed CIN2 + and women with confirmed CIN2 +. hrHPVpositive women were classified as infected with (I) HPVI6, (2) HPVI8 without presence of HPV I6, (3) HPV3I or 45 without HPV I 6 nor HPV I8, or (4) none of the HPV types 16, 18, 31, or 45.

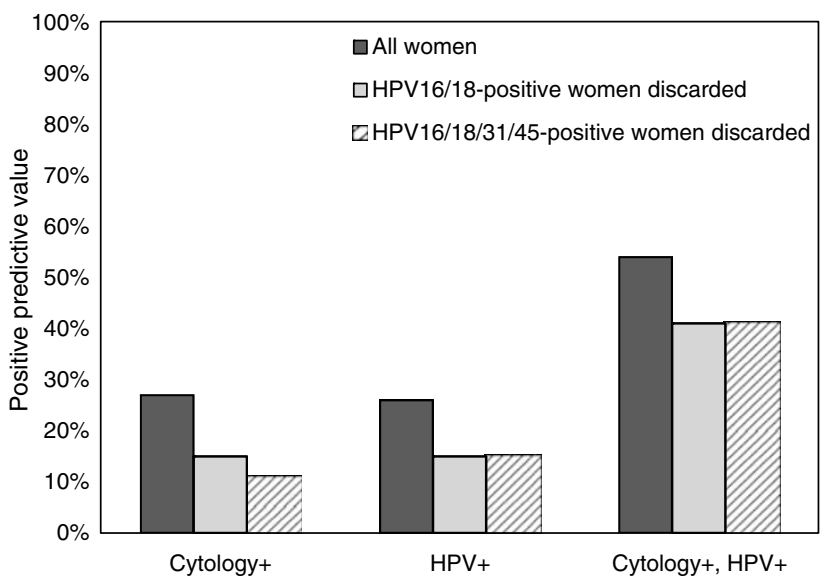

Figure 5 The positive predictive values for detection of $\mathrm{CIN} 2+$ lesions in women aged 29-6I years eligible for routine screening in case screening had been carried out by means of cytology, HPV DNA testing, or both. Results are shown for the current data set (black bars), when excluding HPVI6//8-positive infections from the data (grey bars), and when excluding HPVI6/|8/3|/45-positive infections (striped bars).

hrHPV test, and double-positive tests decreased to 15,15 , and $41 \%$, respectively. When excluding all HPV16/18/31/45-positive infections, the positive predictive values became 11,15 , and $41 \%$, respectively.

\section{DISCUSSION}

In this study, data from a cohort of 45362 Dutch women between 18 and 65 years of age were used to estimate the relationship between type-specific hrHPV prevalence and age in the Netherlands. We have shown that the prevalence of hrHPV peaks between 20 and 25 years, and we estimated the peak prevalence at $24 \%$. Furthermore, we have shown that the proportion of HPV16 infections in hrHPV-positive smears decreases with age. We estimated that around $65 \%$ of hrHPV-positive women with a confirmed CIN2 + have an HPV16 and/or 18 infection. This indicates that non-HPV16/18 infections remain important with HPV16/18 vaccination.

The data set consisted of women aged 29-61 years eligible for routine screening and women younger than 29 or older than 61 years outside the routine screening age range. The hrHPV prevalence in women outside the routine screening age range may be biased because they visited their GP on own initiative. However, comparison of the HPV prevalence in opportunistically screened women aged 27 or 28 years to screening-eligible women aged 29 or 30 years did not show a trend shift in HPV prevalence (data not shown). Moreover, we observed an overall hrHPV prevalence of $21 \%$ in the age group 18-24 years, which is consistent with the international literature (Winer et al, 2003; Manhart et al, 2006; Cuzick et al, 2006).

Regarding the relationship between the hrHPV prevalence and age, we observed high prevalences at young age, and a subsequent decrease until 45 years of age. Above 45 years, the hrHPV prevalence reached a plateau level. The decrease in hrHPV prevalence was also observed for each hrHPV type separately. This decrease may be related to several factors that have been discussed in detail in the literature (Jacobs et al, 2000b; Castle et al, 2006). The observed relationship between hrHPV prevalence and age is in agreement with data from several European and North American studies (Jacobs et al, 2000a; Sellors et al, 2000; de Sanjose et al, 2003; Castle et al, 2005; Ronco et al, 2005; Manhart et al, 2006). In our large data set, we did not find a second hrHPV peak at age beyond 45 years as detected in several Latin American countries (Franceschi et al, 2006). Within hrHPVpositive women, HPV16 and HPV31 were the most prevalent types. Both types were also the most prevalent ones in an earlier pooled analysis of data from three European countries (Clifford et al, 2005).

A remarkable finding of this study is the negative association between the prevalence of HPV16 in hrHPV infections and age. A similar negative association was found in women eligible for routine screening with a confirmed CIN2 + lesion. For the other HPV types in hrHPV infections, associations with age were zero or minor. Although, in absolute sense, the decrease in HPV16 infections with age was small, we think that our observation reveals an interesting effect of cervical screening. HPV16 infections have a higher chance of progressing to cervical lesions than other infections; therefore, HPV16 infections also have a higher chance of being detected during the first round of routine cytological screening. In that case, the proportion of HPV16 infections will decrease at older age because the lesions containing the HPV16 infection have been eradicated.

From our results, it can be inferred that the maximum reductions in hrHPV infections and in CIN2 + lesions that can be achieved by prophylactic HPV16/18 vaccination would be about 42 and $65 \%$, respectively. When an HPV16/18 vaccine would also offer full cross-protection against types 31 and 45 (Harper et al, 2006), the maximum reductions in hrHPV infections and in CIN2 + lesions would be about 60 and $76 \%$, respectively. In reality, the reductions will be smaller because women with an HPV16/18/31/45-positive infection may be co-infected with another type. To summarise, screening remains necessary to detect at least $20-30 \%$ of the CIN2 + cases that cannot be prevented by prophylactic vaccination.

Implementation of mass vaccination may affect screening programmes. The primary aim of screening in a vaccinated population is to detect (pre-)cancerous lesions caused by nonHPV16/18 infections. We showed that the positive predictive values of cytology and HPV testing decrease when HPV16/18 infections have been removed from the data. The main reason for this is that HPV16 has a relatively high prevalence in hrHPV-positive women with confirmed high-grade CIN. This diminishes the effect of cervical screening on the incidence of cervical cancer and may lead to a discussion about the 
cost-effectiveness of cervical screening in vaccinated women. Although formal modelling analyses are required to evaluate the cost-effectiveness of screening strategies with vaccination, changes in the length of the screening interval and/or the age at which screening starts can be anticipated. A related discussion concerns the choice of the primary screening instrument. Metaanalyses have shown that, HPV DNA testing has a higher sensitivity than cytology (Arbyn et al, 2006; Cuzick et al, 2006) and may be implemented in cervical screening in the future. In this study, we have shown that on removing HPV16/18 infections, the positive predictive values of HPV testing and cytology show similar decreases. However, in a population vaccinated against HPV16/18, additional factors may cause a further degradation of the positive predictive value of cytology (Franco et al, 2006). More specifically, a decreasing prevalence of high-grade CIN may make it increasingly more difficult to differentiate abnormalities in cervical smears. On the other hand, we also computed that a high positive predictive value can still be achieved when cytology is applied only as a triage tool in HPVpositive women. To illustrate, we computed a CIN2 + detection rate of about $40 \%$ in hrHPV-positive women with abnormal

\section{REFERENCES}

Arbyn M, Sasieni P, Meijer CJ, Clavel C, Koliopoulos G, Dillner J (2006) Chapter 9: clinical applications of HPV testing: a summary of metaanalyses. Vaccine 24(Suppl 3): S78-S89

Bulk S, van Kemenade FJ, Rozendaal L, Meijer CJ (2004) The Dutch CISOEA framework for cytology reporting increases efficacy of screening upon standardisation since 1996. J Clin Pathol 57: 388-393

Bulkmans NW, Rozendaal L, Snijders PJ, Voorhorst FJ, Boeke AJ, Zandwijken GR, van Kemenade FJ, Verheijen RH, Groningen K, Boon ME, Keuning HJ, van Ballegooijen M, van den Brule AJ, Meijer CJ (2004) POBASCAM, a population-based randomized controlled trial for implementation of high-risk HPV testing in cervical screening design, methods and baseline data of 44102 women. Int J Cancer 110: $94-101$

Castle PE, Jeronimo J, Schiffman M, Herrero R, Rodriguez AC, Bratti MC, Hildesheim A, Wacholder S, Long LR, Neve L, Pfeiffer R, Burk RD (2006) Age-related changes of the cervix influence human papillomavirus type distribution. Cancer Res 66: 1218-1224

Castle PE, Schiffman M, Herrero R, Hildesheim A, Rodriguez AC, Bratti MC, Sherman ME, Wacholder S, Tarone R, Burk RD (2005) A prospective study of age trends in cervical human papillomavirus acquisition and persistence in Guanacaste, Costa Rica. J Infect Dis 191: $1808-1816$

Clifford GM, Gallus S, Herrero R, Munoz N, Snijders PJ, Vaccarella S, Anh PT, Ferreccio C, Hieu NT, Matos E, Molano M, Rajkumar R, Ronco G, de Sanjose S, Shin HR, Sukvirach S, Thomas JO, Tunsakul S, Meijer CI, Franceschi S (2005) Worldwide distribution of human papillomavirus types in cytologically normal women in the International Agency for Research on Cancer HPV prevalence surveys: a pooled analysis. Lancet 366: $991-998$

Cuzick J, Clavel C, Petry KU, Meijer CJ, Hoyer H, Ratnam S, Szarewski A, Birembaut P, Kulasingam S, Sasieni P, Iftner T (2006) Overview of the European and North American studies on HPV testing in primary cervical cancer screening. Int J Cancer 119: 1095-1101

de Sanjose S, Almirall R, Lloveras B, Font R, Diaz M, Munoz N, Catala I, Meijer CJ, Snijders PJ, Herrero R, Bosch FX (2003) Cervical human papillomavirus infection in the female population in Barcelona, Spain. Sex Transm Dis 30: 788-793

Franceschi S, Herrero R, Clifford GM, Snijders PJ, Arslan A, Anh PT, Bosch FX, Ferreccio C, Hieu NT, Lazcano-Ponce E, Matos E, Molano M, Qiao YL, Rajkumar R, Ronco G, de SS, Shin HR, Sukvirach S, Thomas JO, Meijer CJ, Munoz N (2006) Variations in the age-specific curves of human papillomavirus prevalence in women worldwide. Int J Cancer 119: $2677-2684$

Franco EL, Cuzick J, Hildesheim A, de SS (2006) Chapter 20: Issues in planning cervical cancer screening in the era of HPV vaccination. Vaccine 24(Suppl 3): S171-S177 cytology after removing all HPV16/18/31/45 infections. Such preliminary calculations indicate that screening in women vaccinated for HPV16/18 can still be performed with maintenance of the CIN2 + detection rate after referral. However, screening algorithms must be carefully re-evaluated with regard to the choice of the primary and secondary screening modality and length of the screening interval.

\section{ACKNOWLEDGEMENTS}

This study was funded by ZON, Zorg Onderzoek Nederland (The Netherlands Organisation for Health Research and Development; Grant 30-05220). We gratefully acknowledge the work of the 242 GPs and their assistants and thank the research analysts of the Unit Molecular Pathology, VU University Medical Centre, Amsterdam, for hrHPV testing and HPV typing and the cytotechnologists (Spaarne Ziekenhuis, Heemstede; Stichting PA Laboratorium Kennemerland, Haarlem; Leiden Cytology and Pathology Laboratory, Leiden; Unit Cytopathology, VU University Medical Centre, Amsterdam) for cytological testing.
Harper DM, Franco EL, Wheeler CM, Moscicki AB, Romanowski B, RoteliMartins CM, Jenkins D, Schuind A, Costa Clemens SA, Dubin G (2006) Sustained efficacy up to 4.5 years of a bivalent L1 virus-like particle vaccine against human papillomavirus types 16 and 18: follow-up from a randomised control trial. Lancet 367: $1247-1255$

Jacobs MV, Snijders PJ, van Den Brule AJ, Helmerhorst TJ, Meijer CJ, Walboomers JM (1997) A general primer GP5+/GP6(+)-mediated PCRenzyme immunoassay method for rapid detection of 14 high-risk and 6 low-risk human papillomavirus genotypes in cervical scrapings. J Clin Microbiol 35: $791-795$

Jacobs MV, Walboomers JM, Snijders PJ, Voorhorst FJ, Verheijen RH, Fransen-Daalmeijer N, Meijer CJ (2000a) Distribution of 37 mucosotropic HPV types in women with cytologically normal cervical smears: the age-related patterns for high-risk and low-risk types. Int J Cancer 87: $221-227$

Jacobs MV, Walboomers JM, Snijders PJ, Voorhorst FJ, Verheijen RH, Fransen-Daalmeijer N, Meijer CJ (2000b) Distribution of 37 mucosotropic HPV types in women with cytologically normal cervical smears: the age-related patterns for high-risk and low-risk types. Int J Cancer 87: $221-227$

Koutsky LA, Ault KA, Wheeler CM, Brown DR, Barr E, Alvarez FB, Chiacchierini LM, Jansen KU (2002) A controlled trial of a human papillomavirus type 16 vaccine. $N$ Engl J Med 347: 1645-1651

Manhart LE, Holmes KK, Koutsky LA, Wood TR, Kenney DL, Feng Q, Kiviat NB (2006) Human papillomavirus infection among sexually active young women in the United States: implications for developing a vaccination strategy. Sex Transm Dis 33: 502-508

Munoz N, Bosch FX, de Sanjose S, Herrero R, Castellsague X, Shah KV, Snijders PJ, Meijer CJ (2003) Epidemiologic classification of human papillomavirus types associated with cervical cancer. $N$ Engl J Med 348: $518-527$

Ronco G, Ghisetti V, Segnan N, Snijders PJ, Gillio-Tos A, Meijer CJ, Merletti F, Franceschi S (2005) Prevalence of human papillomavirus infection in women in Turin, Italy. Eur J Cancer 41: 297-305

Sellors JW, Mahony JB, Kaczorowski J, Lytwyn A, Bangura H, Chong S, Lorincz A, Dalby DM, Janjusevic V, Keller JL (2000) Prevalence and predictors of human papillomavirus infection in women in Ontario, Canada. Survey of HPV in Ontario Women (SHOW) Group. Can Med Assoc J 163: 503-508

van den Brule AJ, Pol R, Fransen-Daalmeijer N, Schouls LM, Meijer CJ, Snijders PJ (2002) GP5+/6+ PCR followed by reverse line blot analysis enables rapid and high-throughput identification of human papillomavirus genotypes. J Clin Microbiol 40: 779-787

Villa LL, Costa RL, Petta CA, Andrade RP, Paavonen J, Iversen OE, Olsson SE, Hoye J, Steinwall M, Riis-Johannessen G, ndersson-Ellstrom A, Elfgren K, Krogh G, Lehtinen M, Malm C, Tamms GM, Giacoletti K, 
Lupinacci L, Railkar R, Taddeo FJ, Bryan J, Esser MT, Sings HL, Saah AJ, Barr E (2006) High sustained efficacy of a prophylactic quadrivalent human papillomavirus types 6/11/16/18 L1 virus-like particle vaccine through 5 years of follow-up. $\mathrm{Br} J$ Cancer 95: $1459-1466$
Winer RL, Lee SK, Hughes JP, Adam DE, Kiviat NB, Koutsky LA (2003) Genital human papillomavirus infection: incidence and risk factors in a cohort of female university students. Am J Epidemiol 157: $218-226$

Wold S (1974) Spline functions in data-analysis. Technometrics 16: 1-11. 